\title{
Parathyroid adenoma: a case report
}

\author{
Shehnaz R. Kantharia ${ }^{1 *}$, Rajesh A. Kantharia ${ }^{2}$, Pradeep K. Reddy ${ }^{2}$, Yogesh M. Mistry ${ }^{3}$
}

\begin{abstract}
${ }^{1}$ Department of ENT, Head and Neck Surgery, ${ }^{2}$ Department of Head and Neck Onco Surgery, ${ }^{3}$ Department of Pathology, Kailash Cancer Hospital, Muni Seva Ashram, Goraj, Baroda, Gujarat, India
\end{abstract}

Received: 28 January 2020

Revised: 17 June 2020

Accepted: 30 June 2020

\section{*Correspondence:}

Dr. Shehnaz R. Kantharia,

E-mail: shehnaz.kantharia@greenashram.org

Copyright: (C) the author(s), publisher and licensee Medip Academy. This is an open-access article distributed under the terms of the Creative Commons Attribution Non-Commercial License, which permits unrestricted non-commercial use, distribution, and reproduction in any medium, provided the original work is properly cited.

\begin{abstract}
The incidence of primary hyperparathyroidism in India is 2.5/1000 individuals. Primary hyperparathyroidism can be caused by a non-cancerous parathyroid adenoma, hyperplasia or rarely by parathyroid carcinoma. Most of these patients have few or no symptoms. Correct diagnosis can be reached by clinical settings, biochemical and radiological tests and final confirmation by histopathology of the specimen.
\end{abstract}

Keywords: Parathyroid adenoma, Hyperparathyroidism, Parathyroid hormone, 99mTc-Sestamibi scan

\section{INTRODUCTION}

Primary hyperparathyroidism is part of a spectrum of parathyroid proliferative disorder that includes parathyroid adenoma, parathyroid hyperplasia, and parathyroid carcinoma. $80-85 \%$ of cases accounts for parathyroid adenoma, $15 \%$ with hyperplasia and $0.5-1 \%$ carcinoma respectively. Parathyroid adenoma is a benign neoplasm, commonly sporadic, and most often seen in women than men (3:1). It can occur at any age, although most occur between 50-60 years of age. Patients typically present with evidence of primary hyperparathyroidism with elevated serum calcium levels and elevated serum parathyroid hormone levels. ${ }^{1}$

\section{CASE REPORT}

A 33-year-old male patient presented to a general physician with a history of gradual weight loss, over a period of 8 months from $50 \mathrm{~kg}$ to $36 \mathrm{~kg}$. On Serological investigation patient's serum creatinine was elevated, 2.6 $\mathrm{mg} / \mathrm{dl}$ (reference range 0.4-1.4 mg/dl). Ultrasonography of kidney-ureter-bladder was performed which showed right upper ureteric calculi causing mild hydronephrosis and hydroureter. Patient was referred to Urologist and underwent Holmium laser lithotripsy and DJ stenting. The patient was referred to an Endocrinologist, serum parathyroid hormone (PTH) investigation was done which showed a marked increase level of $339.2 \mathrm{pg} / \mathrm{ml}$ suggestive of primary hyperparathyroidism. Patient then underwent a 99mTc-sestamibi scan (MIBI). The scan showed tracer activity in the lower pole region of thyroid bed representing parathyroid adenoma involving the lower left group of parathyroid gland.

Washout images showed complete wash out of the tracer from the thyroid gland with a residual activity in the lower pole region of thyroid gland.

The patient was then referred to us at the ENT and Head and Neck Surgery Department of Kailash Cancer Hospital and Research Centre, Goraj. A complete ENT examination was conducted. Hopkin's Examination showed bilateral mobile vocal cords. Neck examination was normal and there was no neck swelling or enlarged cervical lymph nodes. An ultrasound of the neck revealed a well-defined hyperechoic nodule of size $(0.8 \times 0.8 \times 1.7$ $\mathrm{cm})$ in thyroid bed postero-inferior to left lower pole of thyroid that was suggestive of a parathyroid adenoma. 
Both the lobes of thyroid appeared normal in size, shape, echotexture and vascularity. There was no evidence of abnormally enlarged cervical lymph nodes. USG of the abdomen revealed changes of medullary nephrocalcinosis involving both kidneys and also showed presence of multiple intraductal/ intraglandular pancreatic stones. Preoperative S. calcium and ionized S. calcium were within normal limits, which along with increased parathyroid hormone level established a diagnosis of normocalcemic primary hyper parathyroidism.
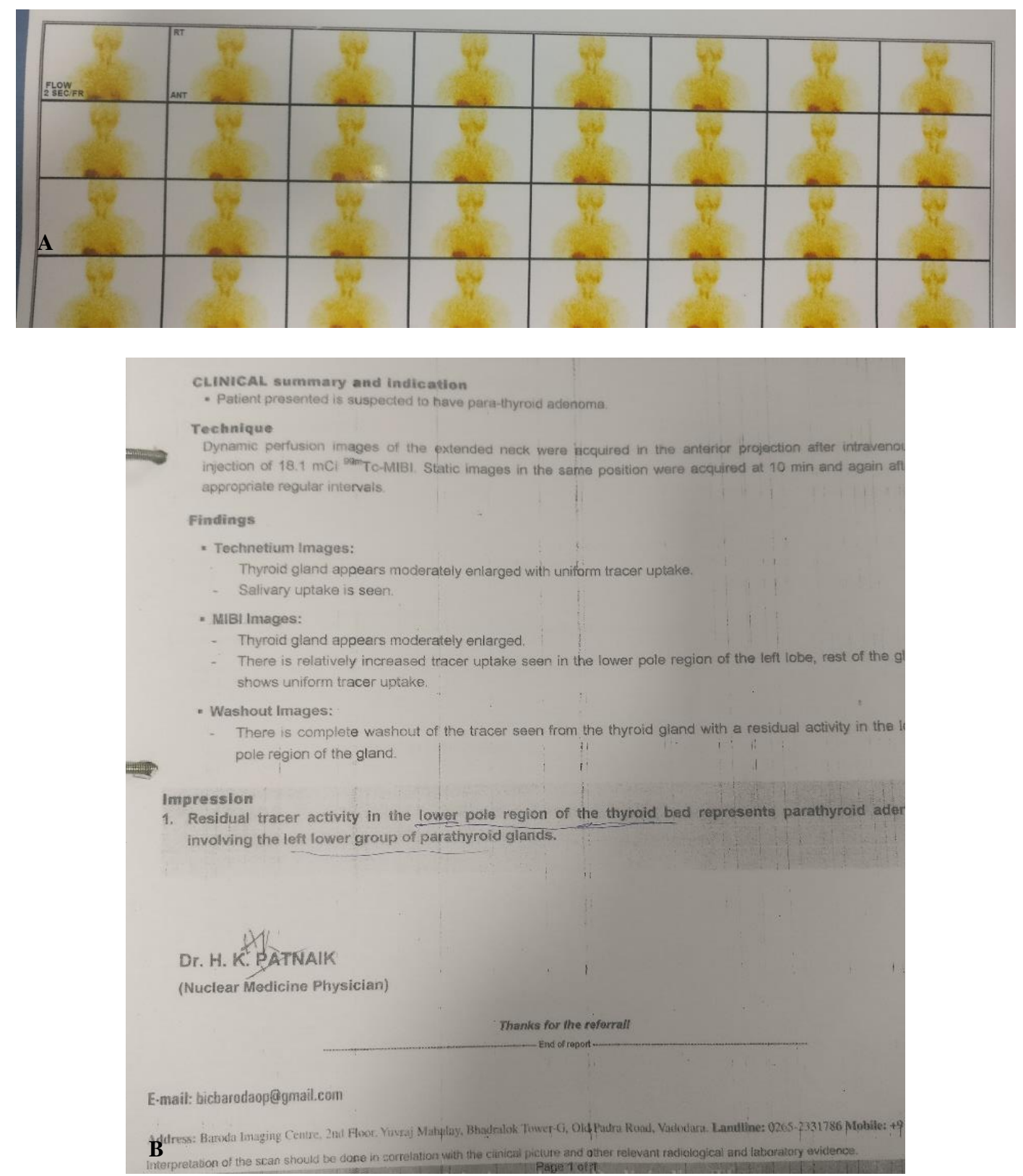

\section{Figure 1: (A) Sestamibi scan showing increased tracer uptake in lower pole region of left lobe of thyroid} gland and (B) report of 99m Tc- MIBI.

Patient was taken for minimally invasive left parathyroidectomy under general anesthesia. A $2 \mathrm{~cm}$ horizontal incision was made in the left lower Neck. The left lobe of thyroid was identified and identification of the enlarged left lower parathyroid was done which was posteroinferior to the left thyroid lobe. The preoperative $99 \mathrm{~m}$ Tc- Sestamibi scintigraphy had localized the enlarged left lower parathyroid as the cause of primary hyperthyroidism. The left inferior parathyroid gland was anterior to the plane of the recurrent laryngeal nerve and was found medial and anterior to the intersection of the recurrent laryngeal nerve and the inferior thyroid artery. With gentle retraction, the enlarged inferior parathyroid gland was pulled up into the neck and removed. The specimen was immediately sent for frozen section. The parathyroid adenoma diagnosis was confirmed intra- 
operatively by the frozen section. The recurrent laryngeal nerve was identified in its normal course in the tracheoesophageal groove and showed no signs of compression from the enlarged parathyroid gland. Level
6 neck dissection was not required as the excised enlarged parathyroid on frozen section confirmed the diagnosis of parathyroid adenoma.
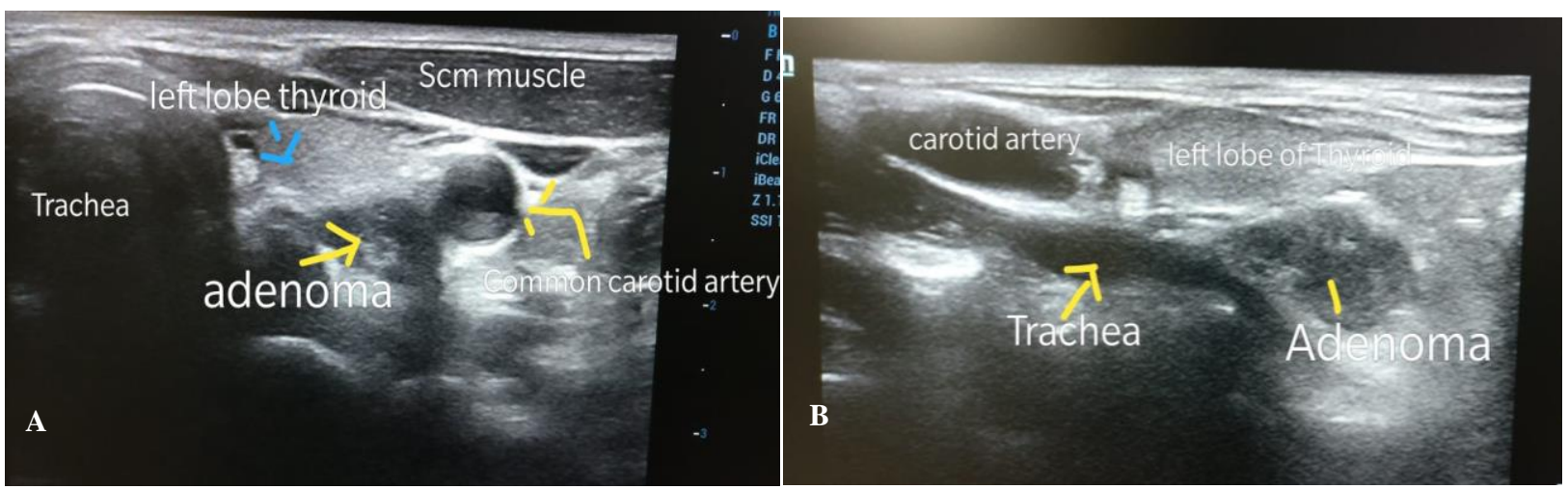

Figure 2: (A) USG image axial section showing well defined hypoechoic nodule in thyroid bed posteroinferior to left lower pole of thyroid and (B) oblique sagittal USG image showing relation of the hypoechoic nodule to trachea.
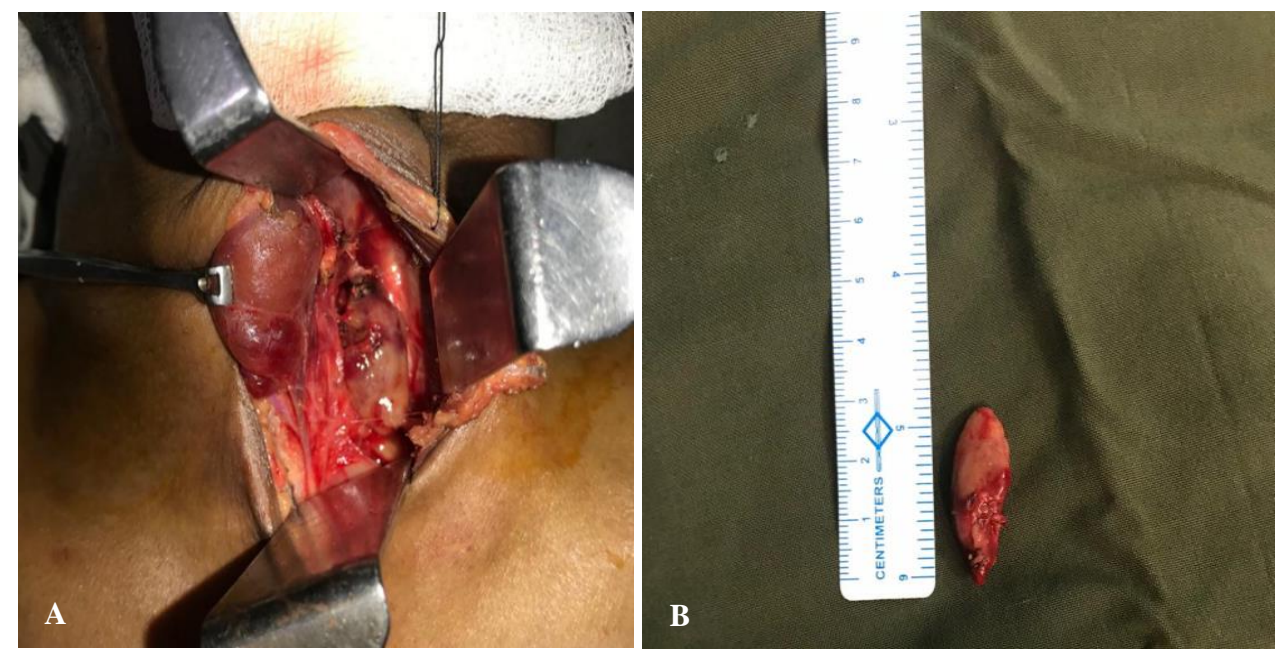

Figure 3: (A) Light brown to tan colored left enlarged parathyroid gland lying posteroinferior to the left thyroid lobe and $(B)$ the excised left parathyroid adenoma measuring $2.9 \times 1.3 \times 0.7 \mathrm{~cm}$.
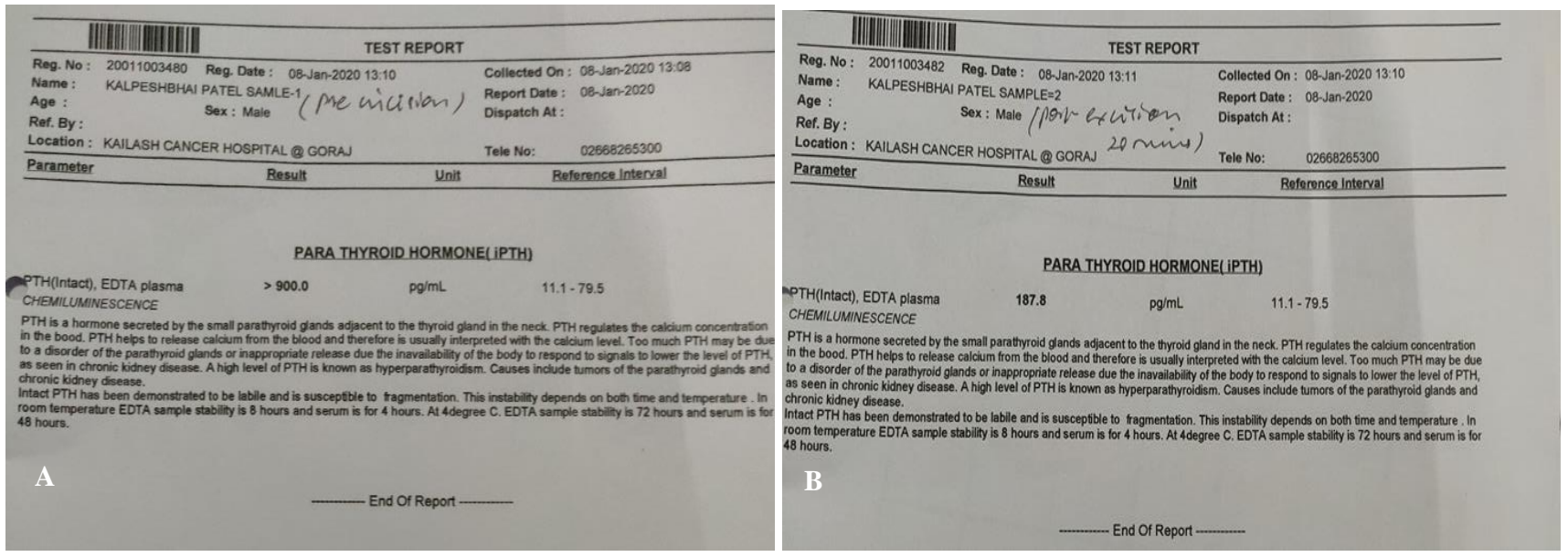

Figure 4: (A) Preoperative parathyroid hormone test (prior to incision) was $>900 \mathrm{pg} / \mathrm{ml}$ and $($ B) intraoperative PTH levels 20 minutes after excision of left enlarged parathyroid was $187.8 \mathrm{pg} / \mathrm{ml}$, an $80 \%$ reduction as compared to pre excisin levels. 
Nodule shows internal vascularity with rim of peripheral vascularity. Laterally it lies in close relation to the common carotid artery.

The preoperative parathyroid hormone level was $>900$ $\mathrm{pg} / \mathrm{ml}$. and 20 minutes post tumor excision the Parathyroid hormone levels were checked which was $187.8 \mathrm{pg} / \mathrm{ml}$, an $80 \%$ fall in PTH levels suggestive of successful tumor excision. Final histopathology report was consistent with parathyroid adenoma. Post-operative period was uneventful, patient had normal voice with bilateral mobile vocal cords and patient was discharged on fourth post-operative day.
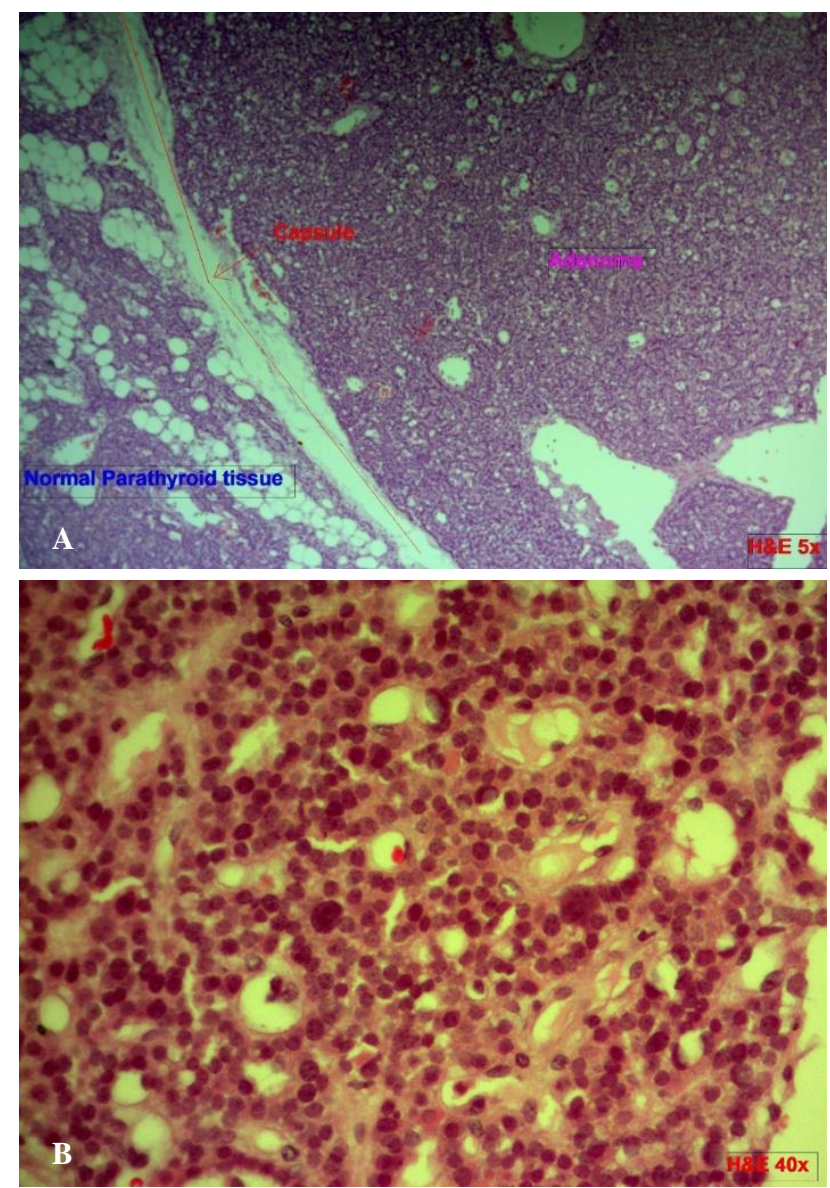

Figure 5: (A) (H\&E; amp 5X): Scanner view section shows thin capsule separating adenoma from normal parathyroid gland and (B) (H\& E; amp 40X): high power view section shows closely packed microfollicles lined by chief cells having monomorphic round nuclei with fine chromatin. Occasional cells with 'neuroendocrine type' nuclear atypia also seen.

\section{DISCUSSION}

Parathyroid adenoma is a relatively rare condition. It has been the major cause of hyperparathyroidism, which occurs when the normal feedback control by serum calcium is disturbed or there is an increased production of PTH. Hyperparathyroidism primarily manifests without symptoms, parathyroid adenomas many a times are insidious, and the only way it can be confirmed is by determination of serum PTH and calcium concentration. Many a times patient gives a pervious history of renal calculus disease, either nephrocalcinosis, or renal or ureteral lithiasis, bone diseases with definite bone involvement, osteoporosis, subperiosteal resorption, osteitis fibrosa, or a combination of renal calculus and bone disease..$^{5-7}$

Normal parathyroid glands are too small to be detected on imaging (usually $5 \times 4 \times 1 \mathrm{~mm}$ ), but parathyroid disease typically results in enlargement of the glands allowing for visualization. Sonography and $99 \mathrm{mTc}$ preoperative sestamibi (MIBI) scan are the primary imaging modalities utilized for the visualization of diseased glands. MIBI scan is approximately $90 \%$ sensitive for localizing a parathyroid adenoma. ${ }^{2}$

Ultrasonography is the first-line method to be used because it is non-invasive, convenient and an inexpensive investigation. Parathyroid adenomas are nearly always homogeneously hypoechoic to the overlying thyroid gland on gray-scale imaging and are commonly detected using gray-scale imaging alone when they are larger than $1 \mathrm{~cm}$ in diameter. Color and power doppler imaging commonly shows a characteristic extrathyroidal feeding vessel (typically a branch off the inferior thyroidal artery), which enters the parathyroid gland at one of the poles. ${ }^{3}$

99mTc-MIBI t61 can be considered to be the first choice in diagnosing hyperparathyroidism as this examination produce a valuable data in locating the site of a tumor before operation. Sestamibi is taken up by both the thyroid and parathyroid glands, but adenomatous and hyperplastic parathyroid tissue shows more avid uptake of the radiotracer and often retains the radiotracer longer than adjacent thyroid tissue..$^{8-10}$

Minimally invasive parathyroidectomy is now most commonly used as the surgical treatment for primary hyperparathyroidism compared to the traditional bilateral neck exploration. Intra operative PTH monitoring is most useful as an adjunct to preoperative imaging allowing for more focused operations to be performed. The use of intra operative PTH monitoring can provide vital information within minutes to help determine the extent of surgical treatment required to be considered optimum. Success is defined using the Miami criteria: a fall in PTH level of $>50 \%$ at $10 \mathrm{~min}$ post-excision compared to baseline (pre-operative). ${ }^{4,11-13}$

\section{CONCLUSION}

Parathyroidectomy stands as the main stay in management of primary hyperparathyroidism due to parathyroid adenoma. Once the biochemical and radiological investigation confirms the presence of parathyroid adenoma, surgical excision is the primary and 
only modality of treatment. Post parathyroidectomy, Parathyroid hormone levels returning to normal within 10 mins of surgery is a conclusive evidence of successful excision of the parathyroid adenoma.

Funding: No funding sources

Conflict of interest: None declared

Ethical approval: Not required

\section{REFERENCES}

1. Wieneke JA, Smith A. Parathyroid adenoma. Head Neck Pathol. 2008;2(4):305-8.

2. Rachkov IV, Georgiev Y. Parathyroid adenoma: case report Trakia J Sci. 2012;10(2):235-9.

3. Johnson NA, Tublin ME, Ogilvie JB. Parathyroid imaging: technique and role in the preoperative evaluation of primary hyperparathyroidism. AJR Am J Roentgenol. 2007;188(6):1706-15.

4. Khan AA, Khatun Y, Walker A, Jimeno J, Hubbard JG. Role of intraoperative PTH monitoring and surgical approach in primary hyperparathyroidism. Ann Med Surg (Lond). 2015;4(3):301-5.

5. Lori A Erickson. Atlas of Endocrine Pathology: Atlas of Anatomic Pathology. Springer; 2014: 121130.

6. Clark PB, Perrier ND, Morton KA.Detection of an intrathymic parathyroid adenoma using singlephoton emissionC T99mTc sestamibi scintigraphy and CT. AIR Am J Roentgenol. 2005;184(3):6-8.

7. Smith JR, Oates ME. Radiolonuclide imaging of parathyroid glands: patterns pearls, and pitfalls. Radiographics 2004;24:1101-15.
8. Wolf RJ, Cronan JJ, Monchik JM. Color Dopple sonography: an adjunctive technique in assessment of parathyroid adenomas. J Ultrasound Med 1994;13:303-8.

9. Lane MJ, Desser TS, Weigel RJ, Jeffrey RB Jr. Us of color and power Doppler sonography to identify feeding arteries associated with parathyroid adenomas AJR. 1998;171:819-23.

10. Reeder SB, Desser TS, Weigel RJ, Jeffrey RB. Sonography in primary hyperparathyroidism: review with emphasis on scanning technique. J Ultrasound Med. 2002;21:539-52.

11. Slater A, Gleeson FV. Increased sensitivity and confidence of SPECT over planar imaging in dualphase sestamibi for parathyroid adenoma detection. Clin Nucl Med. 2005;30:1-3.

12. Spanu A, Falchi A, Manca A, Marongiu P, Cossu A, Pisu N, et al. The usefulness of neck pinhole SPECT as a complementary tool to planar scintigraphy in primary and secondary hyperparathyroidism. J Nucl Med. 2004;45:40-8.

13. Lorberboym M, Minski I, Macadziob S, Nikolov G, Schachter P. Incremental diagnostic value of preoperative 99mTc-MIBI SPECT in patients with parathyroid adenoma. J Nucl Med. 2003;44:904-8.

Cite this article as: Kantharia SR, Kantharia RA, Reddy PK, Mistry YM. Parathyroid adenoma: a case report. Int J Otorhinolaryngol Head Neck Surg 2020;6:1533-7. 